\title{
Evolution of convex hypersurfaces by powers of the mean curvature
}

\section{Journal Article}

Author(s):

Schulze, Felix

Publication date:

2005-12

Permanent link:

https://doi.org/10.3929/ethz-b-000031103

Rights / license:

In Copyright - Non-Commercial Use Permitted

Originally published in:

Mathematische Zeitschrift 251(4), https://doi.org/10.1007/s00209-004-0721-5 


\title{
Evolution of convex hypersurfaces by powers of the mean curvature
}

\author{
Felix Schulze \\ Department of Mathematics, ETH Zürich, 8092 Zürich, Switzerland \\ (e-mail: felix@math.ethz.ch)
}

Received: 5 September 2003; in final form: 18 May 2004 /

Published online: 4 October 2005 - (C) Springer-Verlag 2005

Abstract. We study the evolution of a closed, convex hypersurface in $\mathbb{R}^{n+1}$ in direction of its normal vector, where the speed equals a positive power $k$ of the mean curvature. We show that the flow exists on a maximal, finite time interval, and that, approaching the final time, the surfaces contract to a point.

\section{Introduction}

In this paper we investigate the following problem. Let $M^{n}$ be a smooth, compact manifold without boundary, and $F_{0}: M^{n} \rightarrow \mathbb{R}^{n+1}$ a smooth immersion which is convex. We look for a smooth family of immersions $F(\cdot, t): M^{n} \times[0, T) \rightarrow \mathbb{R}^{n+1}$, which satisfies

$(\star) \quad\left\{\begin{aligned} F(\cdot, 0) & =F_{0}(\cdot) \\ \frac{d F}{d t}(\cdot, t) & =-H^{k}(\cdot, t) v(\cdot, t),\end{aligned}\right.$

where $k>0, H$ is the mean curvature and $v$ is the outer unit normal, such that $-H v=\vec{H}$ is the mean curvature vector. Throughout the paper we will call such a flow an $H^{k}$-flow.

For $k=1$ this flow coincides with the well-known mean curvature flow. In [6], Huisken proved that for this flow the surfaces stay convex and contract to a point in finite time. Furthermore, the surfaces become more and more spherical in the process. This behavior has been shown to be true in many cases where convex surfaces flow by speeds equal to a homogeneous degree one function of the principal curvatures. The evolution by the $n$th root of the Gauss curvature was treated

The author was partially supported by a Schweizerische Nationalfonds grant No. 2166743.01 . 
by Chow in [3] and the evolution by the square root of the scalar curvature in [4]. Andrews [1] showed that this behavior extends to a whole class of such speeds.

If one considers flows for which the speed has an arbitrary positive degree of homogenicity, far less is known about the properties of the flow and the limiting shape of the surface. For a speed equal to a positive power $\alpha$ of the Gauss curvature, Tso [11] and Chow [3] have shown that the surfaces contract to points in finite time. Andrews [2] was able to strengthen this result, showing that for $\alpha \in(1 /(n+2), 1 / n)$ the ratio of the biggest to the smallest principal curvature remains bounded and the surfaces converge after blow-up to a smooth limiting surface.

In [13], Urbas studied the expansion of convex hypersurfaces by symmetric functions of the principal curvatures. This was extended independently by Gerhardt [5] and Urbas [12] to the case of star-shaped surfaces. In both cases the speed was assumed to be homogeneous of degree -1 and the surfaces were shown to converge, under appropriate rescaling, to a sphere.

Investigating our initial value problem $(\star)$, we will have to make a further assumption to ensure short-time existence. We require that $H\left(F_{0}(p)\right)>0$, $\forall p \in M^{n}$, otherwise we cannot guarantee uniform parabolicity. In the following, "weakly convex", and "strictly convex", resp., shall be defined as all the eigenvalues of the second fundamental form of the surface being non-negative, and positive, resp. We obtain the following result:

Theorem 1.1. Let $F_{0}: M^{n} \rightarrow \mathbb{R}^{n+1}$ be a smooth immersion, where $H\left(F_{0}\left(M^{n}\right)\right)>0$. Then there exists a unique, smooth solution to the initial value problem ( $\star$ ) on a maximal, finite time interval $[0, T)$. For $k \geqslant 1$ we have the bound $T \geqslant C(k, n)^{-1}$ $\left(\max _{p \in M}|A|(p, 0)\right)^{-(k+1)}$. In the case that

i) $F_{0}\left(M^{n}\right)$ is strictly convex for $0<k<1$,

ii) $F_{0}\left(M^{n}\right)$ is weakly convex for $k \geqslant 1$,

then the surfaces $F\left(M^{n}, t\right)$ are strictly convex for all $t>0$ and they contract for $t \rightarrow T$ to a point in $\mathbb{R}^{n+1}$.

The paper is organized as follows. In section 2 we compute the evolution equations for several geometric quantities. Using these, we deduce some basic properties of the flow in section 3, which don't depend on the initial surface to be convex, and show the lower bound on the maximal existence time. In section 4 we investigate the case of convex surfaces and prove the second part of the above result.

The author would especially like to thank Professor G. Huisken under whose advice this work was carried out.

\section{Evolution equations}

In this section we compute the evolutions of geometric quantities like the induced metric $g_{i j}$, the second fundamental form $h_{i j}$ or equivalently the Weingarten map $W_{p}=\left\{h_{j}^{i}\right\}: T_{p} M^{n} \rightarrow T_{p} M^{n}$ and the mean curvature $H:=h_{i}^{i}=g^{i j} h_{i j}$.

Lemma 2.1. The evolution equations for $g_{i j}, v, h_{j}^{i}$ and $\langle x, v\rangle$, where $x$ denotes the position vector and $\langle\cdot, \cdot\rangle$ the euclidian scalar product, are given by 
i) $\frac{\partial}{\partial t} g_{i j}=-2 H^{k} h_{i j}$

ii) $\frac{\partial}{\partial t} v=k H^{k-1} \nabla H$

iii) $\frac{\partial}{\partial t} h_{j}^{i}=k H^{k-1} \Delta h_{j}^{i}+k(k-1) H^{k-2} \nabla^{i} H \nabla_{j} H-(k-1) H^{k} h_{l}^{i} h_{j}^{l}+k H^{k-1}|A|^{2} h_{j}^{i}$

iv) $\frac{\partial}{\partial t} H=k H^{k-1} \Delta H+k(k-1) H^{k-2}|\nabla H|^{2}+|A|^{2} H^{k}$

v) $\frac{\partial}{\partial t}\langle x, v\rangle=k H^{k-1} \Delta\langle x, v\rangle-(k+1) H^{k}+k H^{k-1}|A|^{2}\langle x, v\rangle$

Proof. All of the above follows from a direct calculation as for example in [6] or [1].

Apart from the mean curvature $H$ it will be helpful to also study the elementary symmetric polynomials

$$
S_{l}(\lambda)=\sum_{1 \leqslant i_{1}<\ldots<i_{l} \leqslant n} \lambda_{i_{1}} \lambda_{i_{2}} \cdot \ldots \cdot \lambda_{i_{l}} \quad \text { for } \quad \lambda=\left(\lambda_{1}, \ldots, \lambda_{n}\right) \in \mathbb{R}^{n}
$$

of the principal curvatures, and their quotients

$$
Q_{l}(\lambda)=\frac{S_{l}(\lambda)}{S_{l-1}(\lambda)} \quad \text { for } \quad \lambda \in \Gamma_{l-1}
$$

where $S_{0} \equiv 1$, and $S_{l} \equiv 0$ if $l>n ; \Gamma_{l}:=\left\{\lambda \in \mathbb{R}^{n} \mid S_{1}(\lambda)>0, \ldots, S_{l}(\lambda)>0\right\}$. We list here some of their properties, and compute their evolution equations. We denote with $S_{l ; i}(\lambda)$ the sum of all terms in $S_{l}$ not containing the factor $\lambda_{i}$.

Lemma 2.2. For all $l \in\{0, \ldots, n\}, i \in\{1, \ldots, n\}$ and $\lambda \in \mathbb{R}^{n}$ the following equalities hold:

$$
\begin{aligned}
\frac{\partial S_{l+1}}{\partial \lambda_{i}}(\lambda) & =S_{l ; i}(\lambda), \\
S_{l+1}(\lambda) & =S_{l+1 ; i}(\lambda)+\lambda_{i} S_{l ; i}(\lambda), \\
\sum_{i=1}^{n} S_{l ; i}(\lambda) & =(n-l) S_{l}(\lambda), \\
\sum_{i=1}^{n} \lambda_{i} S_{l ; i}(\lambda) & =(l+1) S_{l+1}(\lambda), \\
\sum_{i=1}^{n} \lambda_{i}^{2} S_{l ; i}(\lambda) & =S_{1}(\lambda) S_{l+1}(\lambda)-(l+2) S_{l+2}(\lambda) .
\end{aligned}
$$

\section{Lemma 2.3.}

i) $Q_{l+1}$ is concave on $\Gamma_{l}$ for $l \in\{0, \ldots, n-1\}$.

ii) $\frac{\partial}{\partial \lambda_{i}} Q_{l}(\lambda)>0$ on $\Gamma_{l}$ for all $i \in\{1, \ldots, n\}$ and $l \in\{2, \ldots, n\}$.

For proofs of the last two lemmas see for example [8]. The quotients $Q_{l}$ satisfy the following evolution equation:

Lemma 2.4. For $k \geqslant 1$ let $F: M^{n} \times[0, T) \rightarrow \mathbb{R}^{n+1}$ be an $H^{k}$-flow with

$$
S_{l-1}(p, t)>0 \text { for all }(p, t) \in M^{n} \times[0, T),
$$

such that $Q_{l}$ is well-defined. Then

$$
\frac{\partial}{\partial t} Q_{l} \geqslant k H^{k-1} \Delta Q_{l}+H^{k-1}\left(k|A|^{2}-l(k-1) H Q_{l}\right) Q_{l} .
$$


Proof. Using

$$
\frac{\partial}{\partial t} Q_{l}=\frac{\partial Q_{l}}{\partial h_{j}^{i}}\left(\frac{\partial}{\partial t} h_{j}^{i}\right) \text { and } \Delta Q_{l}=\frac{\partial Q_{l}}{\partial b_{j}^{i}} \Delta h_{j}^{i}+\frac{\partial^{2} Q_{l}}{\partial h_{q}^{p} \partial h_{m}^{l}} \nabla^{v} h_{q}^{p} \nabla_{v} h_{m}^{l}
$$

as well as the evolution equation for $h_{j}^{i}$, we get

$$
\begin{aligned}
\frac{\partial}{\partial t} Q_{l}= & k H^{k-1} \Delta Q_{l}-k H^{k-1} \frac{\partial^{2} Q_{l}}{\partial h_{q}^{p} \partial h_{m}^{l}} \nabla^{v} h_{q}^{p} \nabla_{v} h_{m}^{l}+k(k-1) H^{k-2} \frac{\partial Q_{l}}{\partial h_{j}^{i}} \nabla^{i} H \nabla_{j} H \\
& -(k-1) H^{k} \frac{\partial Q_{l}}{\partial h_{j}^{i}} h_{l}^{i} h_{j}^{l}+k H^{k-1}|A|^{2} \frac{\partial Q_{l}}{\partial h_{j}^{i}} h_{j}^{i} .
\end{aligned}
$$

With the aid of Lemma 2.2 the last two terms can be simplified.

$$
\begin{gathered}
\frac{\partial Q_{l}}{\partial h_{j}^{i}} h_{j}^{i}=\sum_{m} \frac{\partial Q_{l}}{\partial \lambda_{m}} \lambda_{m}=\frac{1}{S_{l-1}^{2}}\left(S_{l-1} \sum_{i} S_{l-1, i} \lambda_{i}-S_{l} \sum_{i} S_{l-2, i} \lambda_{i}\right)=Q_{l} \\
\frac{\partial Q_{l}}{\partial h_{j}^{i}} h_{l}^{i} h_{j}^{l}=\sum_{m} \frac{\partial Q_{l}}{\partial \lambda_{m}} \lambda_{m}^{2}=\frac{1}{S_{l-1}^{2}}\left(S_{l-1} \sum_{i} S_{l-1, i} \lambda_{i}^{2}-S_{l} \sum_{i} S_{l-2, i} \lambda_{i}^{2}\right) \\
=-(l+1) \frac{S_{l+1}}{S_{l-1}}+l Q_{l}^{2} .
\end{gathered}
$$

The monotonicity and the concavity of the $Q_{l}$ 's give the desired inequality .

If the surfaces $M_{t}$ are strictly convex we can also study the inverse $W_{p}^{-1}=\left\{b_{j}^{i}\right\}$ : $T_{p} M^{n} \rightarrow T_{p} M^{n}$ of the Weingarten map $W$, i.e. $b_{l}^{i} h_{j}^{l}=\delta_{j}^{i}$. We obtain:

Lemma 2.5. Let $k>0$ and $M_{t}$ be a flow of strictly convex surfaces. Then

$$
\begin{aligned}
\frac{\partial}{\partial t} b_{j}^{i}= & k H^{k-1} \Delta b_{j}^{i}-2 k H^{k-1} h_{m}^{l} \nabla_{p} b_{l}^{i} \nabla^{p} b_{j}^{m}-k(k-1) H^{k-2}\left(b_{l}^{i} \nabla_{l} H\right)\left(b_{j}^{m} \nabla_{m} H\right) \\
& +(k-1) H^{k} \delta_{j}^{i}-k H^{k-1} b_{j}^{i}|A|^{2} \\
\leqslant & k H^{k-1} \Delta b_{j}^{i}+(k-1) H^{k} \delta_{j}^{i}-k H^{k-1} b_{j}^{i}|A|^{2}
\end{aligned}
$$

Proof. Using $b_{l}^{i} h_{j}^{l}=\delta_{j}^{i}$ we get $\frac{\partial}{\partial t} b_{j}^{i}=-b_{p}^{i} b_{j}^{q} \frac{\partial}{\partial t} h_{q}^{p}$, and $\nabla_{l} b_{j}^{i}=-b_{p}^{i} b_{j}^{q} \nabla_{l} h_{q}^{p}$, which leads to

$$
\Delta b_{j}^{i}=-b_{l}^{i} b_{j}^{m} \Delta h_{m}^{l}+2 h_{m}^{l} \nabla_{p} b_{l}^{i} \nabla^{p} b_{j}^{m} .
$$

Together with Lemma 2.1, this proves the first equality. For $k \geqslant 1$ the inequality follows immediately. To show that also for $0<k<1$ the gradient terms have the desired sign, we have to work a bit more. We want to use the fact that we can write $H^{k}(\lambda)=\left(Q_{n}^{k}(\kappa)\right)^{-1}$, where the $\kappa_{i}$ are the principle radii, i.e. $\kappa_{i}=\lambda_{i}^{-1}$. For general functions $f, g$ satisfying $f\left(h_{j}^{i}\right)=1 / g\left(b_{j}^{i}\right)$ one can now compute that

$$
\frac{\partial^{2} f}{\partial h_{j}^{i} \partial h_{q}^{p}}=\frac{2}{f} \frac{\partial f}{\partial h_{j}^{i}} \frac{\partial f}{\partial h_{q}^{p}}-\frac{1}{g^{2}} \frac{\partial^{2} g}{\partial b_{n}^{m} \partial b_{l}^{k}} b^{m q} b_{n p} b^{j k} b_{i l}-\frac{\partial f}{\partial h^{i p}} b^{j q}-\frac{\partial f}{\partial h_{j q}} b_{i p} .
$$


Applying this we obtain

$$
\begin{aligned}
k(k-1) H^{k-2} \delta_{l}^{m} \delta_{p}^{q}= & \frac{\partial^{2}\left(H^{k}(\lambda)\right)}{\partial h_{l}^{m} \partial h_{p}^{q}} \\
= & 2 k^{2} H^{k-2} \delta_{l}^{m} \delta_{p}^{q}-H^{2 k} \frac{\partial^{2}\left(Q_{n}^{k}\right)}{\partial b_{o}^{n} \partial b_{s}^{r}} b^{n q} b_{o p} b^{r m} b_{s l} \\
& -k H^{k-1} \delta_{l p} b^{m q}-k H^{k-1} \delta^{m q} b_{l p},
\end{aligned}
$$

and by multiplication with $\nabla^{v} h_{l}^{m} \nabla_{w} h_{p}^{q}$ and summation

$$
\begin{aligned}
k(k-1) H^{k-2} \nabla^{v} H \nabla_{w} H= & 2 k^{2} H^{k-2} \nabla^{v} H \nabla_{w} H \\
& -H^{2 k} \frac{\partial^{2}\left(Q_{n}^{k}\right)}{\partial b_{o}^{n} \partial b_{s}^{r}}\left(b^{n q} b_{o p} \nabla_{w} h_{q}^{p}\right)\left(b^{r m} b_{l s} \nabla^{v} h_{m}^{l}\right) \\
& -2 k H^{k-1} b^{m q} \nabla_{w} h_{q}^{p} \nabla^{v} h_{p m} .
\end{aligned}
$$

Using the Codazzi-equations and the concavity of $Q_{n}^{k}(\kappa)$ for $0<k \leqslant 1$, this leads to

$$
-k(k+1) H^{k-2} \nabla^{v} H \nabla_{w} H \geqslant-2 k H^{k-1} b^{m q} \nabla_{p} h_{m}^{v} \nabla^{p} h_{q w},
$$

which finally gives

$$
-k(k-1) H^{k-1}\left(b_{v}^{i} \nabla^{v} H\right)\left(b_{j}^{w} \nabla_{w} H\right) \leqslant 2 k H^{k-1} h_{m}^{l} \nabla_{p} b_{l}^{i} \nabla^{p} b_{j}^{m} .
$$

\section{Basic properties}

We now want to show short-time existence for the initial value problem $\left(\star^{k} H^{k}, k>0\right.$, and study the question of longtime existence. In order to obtain these results, it suffices to demand that the initial surface be only strictly mean convex rather than convex. Hence we require only that $F_{0}: M^{n} \rightarrow \mathbb{R}^{n+1}$ is a smooth, orientable and closed hypersurface with a choice of a normal vector field, such that the initial mean curvature satisfies $H_{0}(p)>\delta>0$ for all $p \in M^{n}$, for some fixed $\delta$. This implies that

$$
\frac{\partial}{\partial \lambda_{i}} H_{0}^{k}(\lambda(p))>0 \text { for all } 1 \leqslant i \leqslant n, p \in M^{n},
$$

which gives with the aid of [7]:

Lemma 3.1. If the mean curvature of $M_{0}$ is strictly positive, then $\left(\star_{H^{k}}\right)$ has a unique solution $F: M^{n} \times[0, T) \rightarrow \mathbb{R}^{n+1}$, at least for a small $T>0$.

Example 3.2. For the evolution of a sphere with radius $R_{0}$ we obtain that

$$
R(t)=\left(R_{0}^{k+1}-(1+k) n^{k} \cdot t\right)^{\frac{1}{k+1}},
$$

which implies a maximal existence time $T=\frac{R_{0}^{k+1}}{n^{k}(1+k)}$.

The evolution equation for $H$ and the maximum principle guarantee that the minimum of $H$ is increasing, i.e. we don't loose the uniform parabolicity of our equation. 


\section{Proposition 3.3.}

$$
H_{\min }(t) \geqslant H_{\min }(0) \cdot\left(1-\frac{k+1}{n} H_{\min }^{k+1}(0) \cdot t\right)^{-\frac{1}{k+1}},
$$

which gives an upper bound on the maximal existence time:

$$
T_{\max } \leqslant \frac{n}{k+1} H_{\min }^{-(k+1)}(0) .
$$

Proof. We apply the inequality $|A|^{2} \geqslant \frac{1}{n} H^{2}$ to the evolution equation of $H$. The estimate then follows immediately by comparison with the ODE

$$
\frac{d}{d t} \varphi=\frac{1}{n} \varphi^{k+2} ; \quad \varphi(0)=H_{\min }(0) .
$$

Remark 3.4. ( $i$ ) The fact that the inequality $H \geqslant \delta$ is maintained under the evolution implies by the strict elliptic maximum principle that an initially embedded hypersurface stays embedded.

(ii) By the strict parabolic maximum principle, as for the mean curvature flow, we have an avoidance principle. If two closed initial hypersurfaces $M_{1}$ and $M_{2}$ are disjoint, then they remain so under the flow. Applying this principle to a big enclosing sphere, we have another upper bound on the maximal existence time.

Proposition 3.5. Let $[0, T)$ be the maximal existence interval of the $H^{k}$-flow $M_{t}$, and $T<\infty$, as well as $H(\cdot, 0) \geqslant \delta_{0}>0$. Then $\limsup _{t \rightarrow T} \max _{M_{t}}|A|^{2}=+\infty$.

Proof. We want to show that under the assumption $\max _{M_{t}}|A|^{2} \leqslant C$, the surfaces $M_{t}$ converge to a smooth limiting surface $M_{T}$. Since $H_{\min }(t)$ is increasing, we have $H_{\min }(T)>0$, and by the shortime existence we get a contradiction to the maximality of $T$.

Assume that $\max _{M_{t}}|A| \leqslant C$ for $0 \leqslant t<T$. Using the evolution equation, and the upper bound for $H$, we can show by integration that

$$
\left|F\left(p, t_{1}\right)-F\left(p, t_{2}\right)\right| \leqslant C\left|t_{1}-t_{2}\right|,
$$

which implies the convergence of $F(\cdot, t)$ to a continuous limiting surface $F(\cdot, T)$. By the bound on $|A|$ the convergence is in $C^{1}$.

For $k \leqslant 1$ the speed $H^{k}$ is concave in $h_{j}^{i}$. Together with the uniform $C^{2}$ bounds, the estimates of Krylov [9], Theorem 2, Chapter 5.5, are applicable and render uniform $C^{2, \alpha}$-bounds. For $k>1$ we study the flow locally, i.e. given by a height function $u$ which satisfies the parabolic PDE

$$
\frac{\partial}{\partial t} u={\sqrt{1+|D u|^{2}}}^{1-k}\left(\left(\delta^{i j}-\frac{D_{i} u D_{j} u}{1+|D u|^{2}}\right) D_{i j} u\right)^{k} .
$$

This operator is not concave in the second derivatives of $u$. With the bound on $|A|$ we can nevertheless check that the conditions for Theorem 2, chapter 5.3 in [9] 
are satisfied, which leads to uniform Hölder-estimates in space and time for $\frac{\partial}{\partial t} u$. Theorem 4 in chapter 5.2 then gives similar Hölder-estimates for $D u$. Because of $H(\cdot, t) \geqslant \delta$ and equation (2) this implies that

$$
v:={\sqrt{1+|D u|^{2}}}^{1-k} \text { and } w:=\left(\left(\delta^{i j}-\frac{D_{i} u D_{j} u}{1+|D u|^{2}}\right) D_{i j} u\right)^{k-1}
$$

are also uniformly Hölder-continuous in space and time. Using this, we can write (2) as a linear, strictly parabolic PDE

$$
\frac{\partial}{\partial t} u-a^{i j} D_{i j} u=0
$$

with coefficients $a^{i j}$ in $C^{\beta}$ in space and time. The interior Schauder estimates, see for example [10], lead to $C^{2, \beta}$-bounds. In both cases, i.e. $k \leqslant 1$ and $k>1$, using again parabolic Schauder estimates, we get a bound on all the higher $C^{l}$-norms.

Remark 3.6. $C^{2, \alpha}$-bounds up to the initial Hypersurface $M_{0}$ also follow from the results of [9]. They depend only on the bound on $|A|$ and the $C^{2, \alpha}$-norm of $M_{0}$.

To show the lower bound on the maximal existence time, i.e. to control the second fundamental form, we will have to study the evolution of $\lambda_{\min }(t):=\min _{M_{t}} \lambda_{i}$. To do this, we define a smooth approximation $\tilde{u}$ to $\max \left(x_{1}, \ldots, x_{n}\right)$ as follows. For $\delta>0$ let

$$
\begin{gathered}
\tilde{u}_{2}\left(x_{1}, x_{2}\right)=\frac{x_{1}+x_{2}}{2}+\sqrt{\left(\frac{x_{1}-x_{2}}{2}\right)^{2}+\delta^{2}} \\
\tilde{u}_{n+1}\left(x_{1}, \ldots, x_{n+1}\right)=\frac{1}{n+1} \sum_{i=1}^{n+1} \tilde{u}_{2}\left(x_{i}, \tilde{u}_{n}\left(x_{1}, \ldots, \hat{x}_{i}, \ldots, x_{n+1}\right)\right), n \geqslant 2 .
\end{gathered}
$$

This approximation has the following properties.

Lemma 3.7. For $\delta>0$ and $n \geqslant 2$,

(i) $\tilde{u}_{n}\left(x_{1}, \ldots, x_{n}\right)$ is smooth, symmetric, monotonically increasing and convex.

(ii) $\frac{\partial \tilde{u}_{n}\left(x_{1}, \ldots, x_{n}\right)}{\partial x_{i}} \leqslant 1$

(iii) $\max \left(x_{1}, \ldots, x_{n}\right) \leqslant \tilde{u}_{n}\left(x_{1}, \ldots, x_{n}\right) \leqslant \max \left(x_{1}, \ldots, x_{n}\right)+(n-1) \delta$

(iv) $\tilde{u}_{n}\left(x_{1}, \ldots, x_{n}\right)-(n-1) \delta \leqslant \sum_{i=1}^{n} \frac{\partial \tilde{u}_{n}\left(x_{1}, \ldots, x_{n}\right)}{\partial x_{i}} \cdot x_{i} \leqslant \tilde{u}_{n}\left(x_{1}, \ldots, x_{n}\right)$

(v) $\sum_{i} \frac{\partial \tilde{u}_{n}}{\partial x_{i}}=1$.

Proof. Direct computation and induction.

Lemma 3.8. Let $F: M^{n} \times[0, \tau) \rightarrow \mathbb{R}^{n+1}$ be an $H^{k}$-flow, $k \geqslant 1$, and $0<H \leqslant C$ on $M^{n} \times[0, \tau)$. For $\lambda_{\min }(t):=\min _{M_{t}} \lambda_{i}(p)$, we have that

$$
\left|\lambda_{\min }(t)\right| \leqslant\left(\left(\max \left\{-\lambda_{\min }(0), C\right\}\right)^{-2}-k C^{\prime}(n) C^{k-1} t\right)^{-\frac{1}{2}},
$$

with $C^{\prime}(n)=8 n\left((n-1)^{2}+1\right)$, as long as the right hand side is finite. 
Proof. For $\beta_{j}^{i}:=-h_{j}^{i}$ we obtain from Lemma 2.1:

$$
\frac{\partial}{\partial t} \beta_{j}^{i} \leqslant k H^{k-1} \Delta \beta_{j}^{i}+(k-1) H^{k} \beta_{l}^{i} \beta_{j}^{l}+k H^{k-1}|A|^{2} \beta_{j}^{i} .
$$

Let $u\left(\beta_{j}^{i}\right)$ be a smooth approximation to $-\min \left(\lambda_{1}, \ldots, \lambda_{n}\right)$ for a $\delta>0$, as defined above. Using

$$
\frac{\partial}{\partial t} u=\frac{\partial u}{\partial \beta_{j}^{i}}\left(\frac{\partial}{\partial t} \beta_{j}^{i}\right) \text { and } \Delta u=\frac{\partial^{2} u}{\partial \beta_{q}^{p} \partial \beta_{m}^{l}} \nabla^{v} \beta_{q}^{p} \nabla_{v} \beta_{m}^{l}+\frac{\partial u}{\partial \beta_{j}^{i}} \Delta \beta_{j}^{i},
$$

as well as the monotonicity of $u$, it follows that, using Lemma 3.7,

$$
\begin{aligned}
\frac{\partial}{\partial t} u \leqslant & k H^{k-1} \Delta u-k H^{k-1} \frac{\partial^{2} u}{\partial \beta_{q}^{p} \partial \beta_{m}^{l}} \nabla^{v} \beta_{q}^{p} \nabla_{v} \beta_{m}^{l} \\
& +(k-1) H^{k} \frac{\partial u}{\partial \beta_{j}^{i}} \beta_{l}^{i} \beta_{j}^{l}+k H^{k-1}|A|^{2} \frac{\partial u}{\partial \beta_{j}^{i}} \beta_{j}^{i} \\
\leqslant & k H^{k-1} \Delta u+(k-1) H^{k}|A|^{2}+k H^{k-1}|A|^{2} u .
\end{aligned}
$$

Since $H>0$, we know that $\lambda_{\max }(p)>0$ and using

$$
\lambda_{\max }(p) \leqslant H-(n-1) \lambda_{\min }(p) \leqslant H+(n-1) u
$$

we can estimate

$$
|A|^{2} \leqslant 2 n\left(H^{2}+(n-1)^{2} u^{2}\right) \leqslant 2 n\left(C^{2}+(n-1)^{2} u^{2}\right) .
$$

If we assume that $u \geqslant C$, we obtain:

$$
\frac{\partial}{\partial t} u \leqslant k H^{k-1} \Delta u+4 n\left((n-1)^{2}+1\right) k C^{k-1} u^{3} .
$$

The estimate then follows by comparing with the ODE and taking the limit $\delta \rightarrow 0$.

Proposition 3.9. Let $F(\cdot, t): M^{n} \times[0, T) \rightarrow \mathbb{R}^{n+1}$ be a smooth $H^{k}$-flow with $k \geqslant 1$, where $T$ is assumed to be maximal. Then we have the lower bound

$$
T \geqslant C(k, n)^{-1}\left(\max _{p \in M}|A|(p, 0)\right)^{-(k+1)},
$$

where $C(k, n)=8(3 k+1)\left(1+(n-1)^{2}\right) n^{(k+3) / 2}$.

Proof. Define $\kappa(t):=\max \left\{-\lambda \min (0), \sup _{M^{n} \times[0, t]} H(p, \tau)\right\}$. Using the bound from the preceeding Lemma and (3), we obtain for $p \in M^{n}$ :

$$
\begin{aligned}
|A|^{2}(p, t) & \leqslant 2 n\left(\kappa^{2}(t)+(n-1)^{2} \lambda_{\min }^{2}(t)\right) \\
& \leqslant 2 n \kappa^{2}(t)+2 n(n-1)^{2}\left(\kappa(t)^{-2}-k C^{\prime}(n) \kappa(t)^{k-1} t\right)^{-1} .
\end{aligned}
$$

We will now assume that

$$
t \leqslant \frac{\kappa(t)^{-(k+1)}}{2 k C^{\prime}(n)}
$$


which is true on a maximal time interval $t \in[0, \varepsilon]$, with $\varepsilon>0$. Thus on this interval the estimate

$$
|A|^{2}(p, t) \leqslant 2 n\left(1+2(n-1)^{2}\right) \kappa(t)^{2} \leqslant C^{\prime}(n) \kappa(t)^{2}
$$

holds. Since $\kappa(t)$ is Lipschitz-continuous in time, we can deduce from the evolution equation for $H$ that for a.e. $t \in[0, \varepsilon]$

$$
\frac{\partial}{\partial t} \kappa(t) \leqslant \max _{M_{t}}|A|^{2}(p) \kappa(t)^{k} \leqslant C^{\prime}(n) \kappa(t)^{k+2},
$$

which leads to

$$
\kappa(t) \leqslant\left(\kappa(0)^{-(k+1)}-(k+1) C^{\prime}(n) t\right)^{-\frac{1}{k+1}} .
$$

We will now show that

$$
\varepsilon \geqslant \frac{1}{(3 k+1) C^{\prime}(n) \kappa(0)^{(k+1)}} .
$$

Assume the contrary. Using this assumption together with (4), we see that

$$
\varepsilon<\frac{1}{(3 k+1) C^{\prime}(n) \kappa(0)^{(k+1)}} \leqslant \frac{\kappa(\varepsilon)^{-(k+1)}}{2 k C^{\prime}(n)},
$$

which is a contradiction to the maximality of $\varepsilon$. Thus (5) holds, which gives with (4):

$$
\begin{aligned}
& \max _{M_{t}} H(p, t) \leqslant \kappa(t) \leqslant\left(\frac{3 k+1}{2 k}\right)^{1 /(k+1)} \kappa(0), \\
& \max _{M_{t}}|A|^{2}(p, t) \leqslant C^{\prime}(n)\left(\frac{3 k+1}{2 k}\right)^{2 /(k+1)} \kappa(0)^{2}
\end{aligned}
$$

for $t \leqslant\left((3 k+1) C^{\prime}(n) \kappa(0)^{(k+1)}\right)^{-1}$. Together with Theorem 3.5 we have thus proven the desired lower bound on the maximal existence time:

$$
T_{\max } \geqslant \frac{1}{(3 k+1) C^{\prime}(n)}(\kappa(0))^{-(k+1)} \geqslant C(k, n)^{-1}\left(\max _{p \in M}|A|(p, 0)\right)^{-(k+1)},
$$

where $C(k, n)=8(3 k+1)\left(1+(n-1)^{2}\right) n^{(k+3) / 2}$.

\section{Convex surfaces}

In this last section we will investigate the $H^{k}$-flow of convex surfaces. We will show that convex surfaces stay convex and that in the case $k \geqslant 1$ they immediately become strictly convex. We will complete the proof of the main theorem by showing that the surfaces contract to a point.

To show that convexity is preserved we again study the evolution of

$$
\lambda_{\min }(t):=\min _{M_{t}} \lambda_{i}
$$


Lemma 4.1. Let $F_{0}\left(M^{n}\right)$ be strictly convex and let $F: M^{n} \times[0, \tau) \rightarrow \mathbb{R}^{n+1}$ be a $H^{k}$-flow, $k>0$. Then all $M_{t}, t \in[0, \tau)$ are strictly convex and $\lambda_{\min }(t)$ is monotonically increasing.

Proof. Heuristically one can see the monotonicity of $\lambda_{\min }(t)$ for $k \geqslant 1$ as follows. Assume in the evolution equation of the Weingarten map, Lemma $2.1 \mathrm{iii}$ ), that we could diagonalize $h_{j}^{i}$ and could study the evolution of $\lambda_{\min }(p, t)$ separately. We would obtain

$$
\frac{\partial}{\partial t} \lambda_{\min }(p, t) \geqslant k H^{k-1} \Delta \lambda_{\min }(p, t)-(k-1) H^{k} \lambda_{\min }^{2}(p, t)+k H^{k-1}|A|^{2} \lambda_{\min }(p, t) .
$$

If we estimate $|A|^{2} \geqslant H \lambda_{\min }(p, t)$ we see that the lowest order terms are nonnegative, and the maximum principle would give the desired result. Since the minimal principal curvature is not a smooth function and for $0<k<1$ the gradient terms have the wrong sign, we have to work a little bit more.

For a fixed $\delta>0$ we choose a smooth approximation $u\left(b_{j}^{i}\right):=\tilde{u}_{n}\left(\kappa_{1}, \ldots, \kappa_{n}\right)$ to $\max \left(\kappa_{1}, \ldots, \kappa_{n}\right)$, as defined in Lemma 3.7, where the $\kappa_{i}$ are the eigenvalues of $b_{j}^{i}$, i.e. $\kappa_{i}=1 / \lambda_{i}$. Using

$$
\frac{\partial}{\partial t} u=\frac{\partial u}{\partial b_{j}^{i}}\left(\frac{\partial}{\partial t} b_{j}^{i}\right) \text { and } \Delta u=\frac{\partial u}{\partial b_{j}^{i}} \Delta b_{j}^{i}+\frac{\partial^{2} u}{\partial b_{q}^{p} \partial b_{m}^{l}} \nabla^{v} b_{q}^{p} \nabla_{v} b_{m}^{l}
$$

as well as the monotonicity of $u$ and Lemma 2.5 we obtain

$$
\begin{aligned}
\frac{\partial}{\partial t} u \leqslant k H^{k-1} \Delta u & -k H^{k-1} \frac{\partial^{2} u}{\partial b_{q}^{p} \partial b_{m}^{l}} \nabla^{v} b_{q}^{p} \nabla_{v} b_{m}^{l} \\
& +(k-1) H^{k} \operatorname{tr}\left(\frac{\partial u}{\partial b_{j}^{i}}\right)-k H^{k-1}|A|^{2} \frac{\partial u}{\partial b_{j}^{i}} b_{j}^{i} .
\end{aligned}
$$

Applying the properties of $\tilde{u}_{n}$ listed above this leads to

$$
\frac{\partial}{\partial t} u \leqslant k H^{k-1} \Delta u+(k-1) H^{k}-k H^{k-1}|A|^{2}(u-(n-1) \delta) .
$$

At a point $(p, t)$ with $u>(n-1) k \delta$ we see that

$$
\frac{\partial}{\partial t} u(p, t)<k H^{k-1} \Delta u(p, t)+(k-1) H^{k-1}\left(\frac{H}{u}-|A|^{2}\right) u(p, t) \leqslant k H^{k-1} \Delta u,
$$

since

$$
\frac{H}{u}(p) \leqslant \frac{H(p)}{\kappa_{\max }(p)}=H(p) \lambda_{\min }(p) \leqslant|A|^{2}(p) .
$$

This gives a contradiction if $u$ attains a first new maximum bigger than $(n-1) k \delta$. The limit $\delta \rightarrow 0$ then proves the claim.

For convex surfaces, i.e. $h_{i j} \geqslant 0$, the full second fundamental form is controlled by its trace: $|A| \leqslant H$. Applying this to the evolution equation for $H$, and comparing with the ODE yields: 
Lemma 4.2. Let $F: M^{n} \times[0, T) \rightarrow \mathbb{R}^{n+1}$ be an $H^{k}$-flow of convex hypersurfaces. Then

$$
|A|(p, t) \leqslant H(p, t) \leqslant\left(H_{\max }(0)^{-(k+1)}-(k+1) t\right)^{-\frac{1}{k+1}} .
$$

Corollary 4.3. Let $F: M^{n} \times[0, T) \rightarrow \mathbb{R}^{n+1}$ be an $H^{k}$-flow and $F_{0}\left(M^{n}\right)$ be weakly convex. Then $F\left(M^{n}, t\right)$ is weakly convex for all $t \in[0, T)$ and $T_{\max } \geqslant$ $\frac{1}{k+1}\left(H_{\max }(0)\right)^{-(k+1)}$.

Proof. We approximate the initial surface $M_{0}$ smoothly by strictly convex surfaces $M_{0}^{i}$, for example using the mean curvature flow. From these surfaces we start $H^{k}$-flows, which by Lemma 4.1 stay convex. By Lemma 4.2 and Proposition 3.5 we have a uniform lower bound $T_{\max }^{i} \geqslant \frac{1}{k+1}\left(H_{\max }(0)\right)^{-(k+1)}$. Using the uniform $C^{2, \alpha}$-estimates from proposition 3.5 , we can extract a convergent subsequence of convex flows, which proves that the original flow also had to be convex.

For $k \geqslant 1$ we could have also applied Hamilton's weak maximum principle for 2-Tensors to the evolution equation of $h_{i j}$ to see that $h_{i j} \geqslant 0$ is conserved.

We now use the quotients $Q_{l}$ to show that for $k \geqslant 1$ weakly convex surfaces immediately become strictly convex.

Proposition 4.4. Let $F_{0}: M^{n} \rightarrow \mathbb{R}^{n+1}$ be a weakly convex hypersurface with $H\left(F_{0}\right) \geqslant \delta>0$, and $F: M^{n} \times[0, T) \rightarrow \mathbb{R}^{n+1}$ the corresponding $H^{k}$-flow with $k \geqslant 1$. Then $M_{t}$ is strictly convex for all $t \in(0, T)$.

Proof. Since $H(p, t)>0$ for all $t \in(0, T), Q_{2}$ is well-defined and non-negative. Because of the bounds on $|A|^{2}$ for $t \in[0, \varepsilon]$,

$$
\left|H^{k-1}\left(k|A|^{2}-2(k-1) H Q_{2}\right)\right| \leqslant C
$$

on this interval. Lemma 2.4 now says that for $v:=e^{C t} Q_{2}$, we have

$$
\frac{\partial}{\partial t} v \geqslant k H^{k-1} \Delta v \text {. }
$$

If we assume that there exists $\left(p_{0}, t_{0}\right) \in M^{n} \times(0, \varepsilon)$ with $Q_{2}\left(p_{0}, t_{0}\right)=0$, then also $v\left(p_{0}, t_{0}\right)=0$. Using the Harnack-inequality in the parabolic case (see i.e. [10]) and the equation above we see that $v \equiv 0$ for all $t \in\left(0, t_{0}\right)$ which gives $Q_{2} \equiv 0$ for all $t \in\left(0, t_{0}\right)$. This is in contradiction to the existence of strictly convex points on $M_{t}$, and so $Q_{2}>0$ on $M^{n} \times(0, T)$. We can iterate this to obtain $Q_{n}>0$ on $M^{n} \times(0, T)$.

The last step towards the proof of Theorem 1.1 is that the flow exists as long as it bounds a non-vanishing volume. Similar estimates, using a lower bound on the support function, were obtained by Tso [11] for the Gauss curvature flow and Andrews [1] for a general class of speeds.

Lemma 4.5. Let $F: M^{n} \times[0, \tau)$ be an $H^{k}$-flow of convex surfaces and for $\delta>0, x_{0} \in \mathbb{R}^{n+1}$, let $B_{\delta}\left(x_{0}\right) \subset V_{t}$ for all $t \in[0, \tau)$, where $V_{t}$ is the volume bounded by $M_{t}$. Then

$$
H(p, t) \leqslant C\left(F_{0}\left(M^{n}\right), \delta, k, n\right) \text { for all }(p, t) \in M^{n} \times[0, \tau) .
$$


Proof. Let $X:=x-x_{0}$ be the position vector field with origin in $x_{0}$. Since all the $M_{t}$ are convex, there is an $\alpha>0, \alpha=\alpha(\delta)$, such that

$$
\langle X, v\rangle-\alpha \geqslant \frac{\alpha}{2}>0 \text { for all }(p, t) \in M^{n} \times[0, \tau) .
$$

So $v:=H^{k} /(\langle X, v\rangle-\alpha)$ is well defined, and satisfies the evolution equation

$$
\begin{aligned}
\frac{\partial}{\partial t} v & =k H^{k-1} \Delta v+\frac{2 k}{H} v \nabla^{i}\langle X, v\rangle \nabla_{i} v+(k+1) v^{2}-\alpha k \frac{|A|^{2}}{H} v^{2} \\
& \leqslant k H^{k-1} \Delta v+\frac{2 k}{H} v \nabla^{i}\langle X, v\rangle \nabla_{i} v+\left((k+1)-\frac{\alpha k}{n} H\right) v^{2}
\end{aligned}
$$

Assume that in $\left(p_{0}, t_{0}\right), v$ attains a big maximum $C \gg 0$ for the first time. Then

$$
H^{k}\left(p_{0}, t_{0}\right) \geqslant C(\langle X, v\rangle-\alpha)\left(p_{0}, t_{0}\right) \geqslant \frac{\alpha C}{2},
$$

which gives a contradiction if

$$
C \geqslant \max \left\{\max _{p \in M} v(p, 0), \frac{2}{\alpha}\left(\frac{n(k+1)}{\alpha k}\right)^{k}\right\} .
$$

Proof of Theorem 1.1. The preceeding Lemma and Proposition 3.5 guarantee that the flow exists as long as it bounds a non-vanishing volume. Because of Lemma 4.1 and Proposition 4.4 all the surfaces are strictly convex for $t>0$. What's more, $\lim _{t \rightarrow T} \lambda_{\min }(t) \geqslant \delta>0$.

Let us assume that there exist two distinct points $x, y \in \mathbb{R}^{n+1}$ with $x, y \in V_{t}$ for all $t \in[0, T), V_{t}$ denoting the volume bounded by $M_{t}$. Then every 2-dimensional plane $Z$ through $x$ and $y$ intersects $M_{t}$ transversally in regular curves $c_{t}^{Z}$. Since $\lambda_{\text {min }}(t)>\frac{\delta}{2}$ there also exists a lower bound $\varepsilon_{0}>0$ on the curvature of the curves $c_{t}^{Z}$. Let $I_{t}:=Z \cap V_{t}$. Since $\mathcal{H}^{n}\left(V_{t}\right) \rightarrow 0$, there exists a $Z$ with $\mathcal{H}^{2}\left(I_{t}\right) \rightarrow 0$. But this contradicts that $x, y \in I_{t}$ and that the curvature of the curves $c_{t}^{Z}$ is uniformly bounded from below.

\section{References}

1. Andrews, B.: Contraction of convex hypersurfaces in Euclidian space. Calc. Var. 2, 151-171 (1994)

2. Andrews, B.: Motion of hypersurfaces by Gauss curvature. Pacific J. Math 195(1), $1-36(2000)$

3. Chow, B.: Deforming convex hypersurfaces by the $n$th root of the Gaussian curvature. J. Diff. Geom. 22, 117-138 (1985)

4. Chow, B.: Deforming hypersurfaces by the square root of the scalar curvature. Invent. Math. 87, 63-82 (1987)

5. Gerhardt, C.: Flow of nonconvex hypersurfaces into spheres. J. Diff. Geom. 32(1), 299-314 (1990) 
6. Huisken, G.: Flow by mean curvature of convex surfaces into spheres. J. Diff. Geom. 20, 237-266 (1984)

7. Huisken, G., Polden, A.: Geometric evolution equations for hypersurfaces. Calc. of Var. and Geom. Evo. Probl., CIME Lectures of Cetraro, Springer, 1996

8. Huisken, G., Sinestrari, C.: Convexity estimates for mean curvature flow and singularities of mean convex surfaces. Acta Math. 183(1), 45-70 (1999)

9. Krylov, N.V.: Nonlinear elliptic and parabolic equations of second order. D. Reidel, 1978

10. Lieberman, G.M.: Second order parabolic differential equations. World Scientific, 1996

11. Tso, K.: Deforming a hypersurface by its Gauss-Kronecker curvature. Comm. Pure Appl. Math. 38, 867-882 (1985)

12. Urbas, J.: On the expansion of starshaped hypersurfaces by symmetric functions of their principal curvatures. Math. Z. 205(3), 355-372 (1990)

13. Urbas, J.: An expansion of convex hypersurfaces. J. Diff. Geom. 33(1), 91-125 (1991) 